\title{
Online GMM Clustering and Mini-Batch- Gradient Descent based Optimization for Industrial IoT 4.0
}

\author{
Messaoud S., Bradai A. IEEE member, and Moulay E.
}

\begin{abstract}
The future fifth generation (5G) Networks are expected to support a huge number of connected devices with a various and multitude service having different Quality of Service $(Q \circ S)$ requirements. Communication in Industry 4.0 is one of the flagships and special applications of the 5G due to the specificity of the industrial environment as well as the variety of its services such as safety communication, robot's communications and machine monitoring. In this context, we propose a new resource allocation for the future industry 4.0 based on SDN and NFV technologies, machine learning tools and the slicing paradigm where each slice of the network is dedicated to a category of services having similar QoS requirement level. The proposed solution ensures the allocation of the resources to the slices depending on their requirement in terms of bandwidth, delay and reliability. Toward this goal, our solution is performed in three main steps: 1) IoT devices assignment to the slices step based on OGMMC algorithm, 2) Inter-slices resources reservations step based on mini batch gradient descent, 3) Intra-slices resources allocations based on the max-utility algorithm. We performed extensive simulations in a realistic industrial scenario using NS3 simulator. Numerical results show the effectiveness of our proposed solution in term of reducing Packet Error Rate (PER), energy consumption, and in terms of increasing the percentage of served devices in delay comparing to the traditional approaches.
\end{abstract}

Index Term s--Network slicing, NFV, Machine Learning, QoS, SDN, Industry 4.0, Inter-slice, Intra-slice.

\section{INTRODUCTION}

$\mathrm{W}$ ith the development of wireless communication technology, the fifth generation $(5 \mathrm{G})$ Wireless Networks are expected to support more than 50 billion of connected devices and machines by 2020 [1]. Therefore, the increased number of generated data and connectivity of smart devices, which are the main driver of the IoT paradigm, are rapidly expanding its benefits in industrial environments. This provides the opportunity to establish industrial cyber-physical system via modern and reliable information exchange technologies and data digitalization [2].

Seifeddine MESSAOUD is with university of Monastir, Laboratory of Electronics and Microelectronics $(\mathrm{E} \mu \mathrm{E})$, Sciences Faculty of Monastir, Monastir 5019, Tunisia (e-mail: seifeddine.messaoud@ fsm.rnu.tn).

Abbas BRADAI is with university of Poitiers, XLIM-CNRS, Bât SP2MI, 11 Bd Marie et Pierre Curie, 86962 Chasseneuil Cedex, France (e-mail: abbas.bradai@univ-poitiers.fr).

Emmanuel MOULAY is with university of Poitiers, XLIM-CNRS, Bât SP2MI, 11 Bd Marie et Pierre Curie, 86962 Chasseneuil Cedex, France (e-mail: emmanue.moulay@univ-poitiers.fr).
This evolution is known by the fourth industrial revolution thanks to the emergence of information and communication technologies, such as cloud computing, IoT, and network softwarization and virtualization, that can be integrated with a multitude of manufacturing processes [3].

Following the industrial revolution and the explosive spread of the IoT paradigm, industrial environment is continuing to support an increasing number of connected objects, such as robot, actuators, machines, and sensors. However, this continuous evolution will bring a radical change of the industrial landscape, allowing new production models and promising commercial opportunities. Furthermore, today's industrial networks have been designed for static manufacturing processes where changes and configurations in the manufacturing workflow require lengthy maintenance operations. In addition, different traffic flows with a multitude and various QoS parameters are transmitted over heterogeneous industrial environments sharing the same physical resources. This will increase cost and data loss, and will not meet the user's QoS requirements. New methodologies and automation mechanisms in the next industrial generation are required to remotely verify the management process, check the QoS, and support new challenges such as improved user's QoS, resource efficiency, and reducing cost.

On the other hand, to support flexibility and sustainability expected in next generation manufacturing processes, industrial networks would be faced with a process of transformation. In this context, the $5 \mathrm{G}$ wireless network system is considered as a key enabler in this new trend by extending the network slicing paradigm to meet the drastic user's requirements, over heterogeneous industrial domains, which represents a revolutionary paradigm for industrial challenges in the coming years.

In this fervent area, network slicing is one of the most powerful solution in industry 4.0 that can bring network drastic improvements and fulfill diverse network requirements based on the unified physical infrastructure and sharing network resources between slices. Supported by network slicing, physical resources can be dynamically reserved and allocated to logical network slices according to the corresponding QoS demands. Under the network slicing mask, Software Defined Networking (SDN) and Network Function Virtualization (NFV) have been widely accepted as promising technologies. These mechanisms can improve decision-making and scalability by efficiently sharing network resources to network slices with different QoS types, managing multiple virtual 
networks, and dynamically configuring parameters [3].

In this context, SDN is defined as a network concept that enables centralized and intelligent control and management that can on-demand modify traffic flows according to industrial application requirements by decoupling data and control traffic. While NFV is the technology that allows the virtual representation and deployment of logical network functions as Virtual Network Functions (VNFs). When these technologies associated with cloud computing, it can provide better solutions to support reliability and latency requirements, which still represents a major challenge of industrial 4.0 applications.

In that regard, great efforts have been carried out to integrate efficiently SDN, NFV, and network slicing basedarchitectures within industrial domains or within another field. Authors in [4] have proposed an industrial network based on SDN mechanism in order to support dynamic production processes. Unlike traditional industrial networks, remarkable energy saving is achieved. A resource allocation method with consideration of interference management was proposed in [5], where different QoS requirements were guaranteed by optimizing jointly power and sub-channel allocation. Authors in [6] have discussed a 5G network architecture scheme based on SDN to allocate physical resources to virtual slices within a local area and to perform scheduling among slices. An end-toend network slicing methodology was proposed by authors of [7] in order to share horizontally physical resources whose main purpose is to create multiple virtual networks that can support industry applications. For interested readers, several architectures based on SDN technology for industrial networks and cyber-physical systems have been proposed recently in [8], [9], [10] and references therein. However, the majority of these proposed works do not deal with real and heterogeneous scenarios with various QoS requirement where different IoT devices or industrial machinery are operating in the same network.

Therefore, unlike previous works, we seek in our paper to extend network slicing benefits in industry 4.0 by considering QoS classes using machine learning tools. The main contributions of this paper are:

1) We propose SDN and NFV based-network slicing architecture for industry 4.0 to meet various services requirements with guaranteed QoS for connected devices;

2)We propose an adaptive and online machine learning algorithm to learn devices requirements and assign each device to the slice that meet its QoS requirements;

3) We propose a machine learning based-dynamic inter-slicing algorithm; to split radio resources and reserve channels to slices based on required throughput;

4)We propose an intra-slicing algorithm in order to allocate dynamically resources on gateways for assigned devices; load and reliability are considered in each gateway.

The leftovers of this paper is organized in five sections. Section II is allocated to the proposed slicing architecture and optimization problem formulation. We introduce in section III the proposed slicing approach. In section IV, we provide a discussion about simulation results and comparisons. Finally, we conclude our paper in section V.

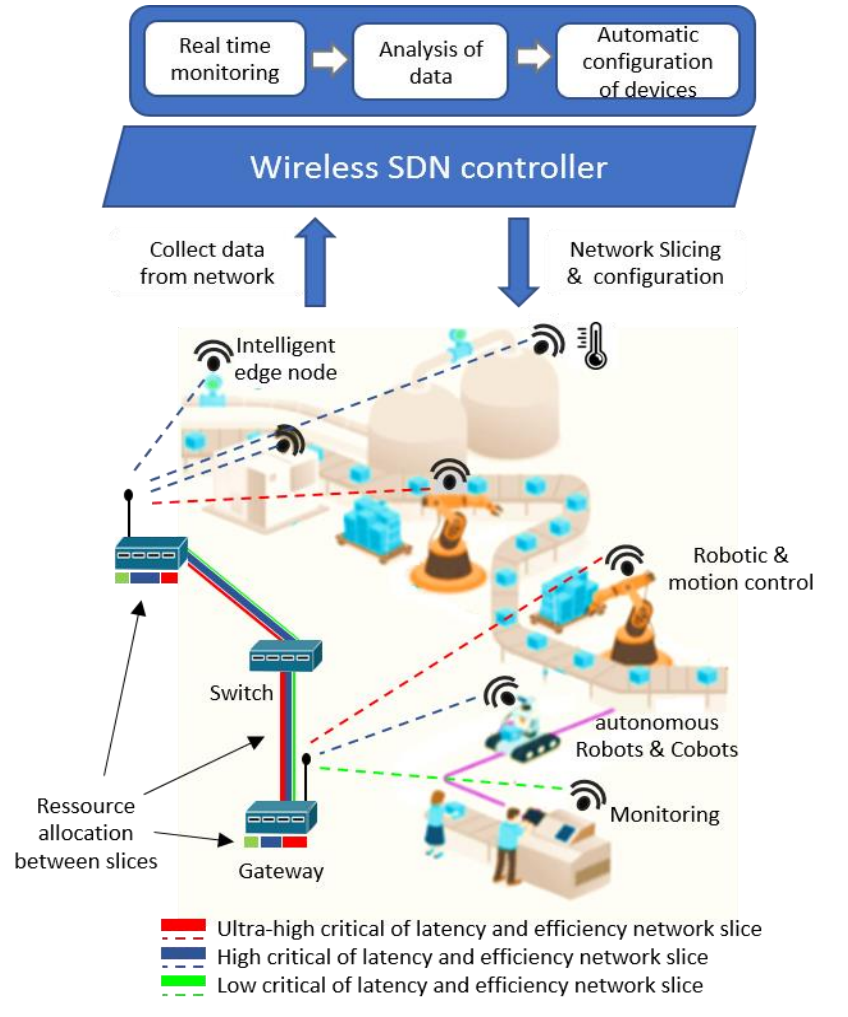

Figure 1: Network Slicing Architecture for industry 4.0

\section{Proposed Network Slicing Architecture And PROBLEM FORMULATION}

\section{A. Proposed Network Slicing Architecture}

The 5G network architecture design should be built on deep consideration of hardware infrastructure, software control, and the interconnectivity between them. The network slicing, which can satisfy multiple service requirements based on the unified physical infrastructure and sharing the same physical resources, is considered as a critical paradigm by providing multiple instances that operate independently for a specific network functions.

The network slicing-based $5 \mathrm{G}$ system architecture is given in Figure 1. The aim of this architecture is to support the creation, control, and management of multiple network slices over factory infrastructures in order to provide high levels of flexibility and scalability and meet QoS requirements for robots, sensors, and actuators in industrial networks.

The infrastructure layer contains all the physical resources needed to perform virtualized industrial processes. We emphasize that the involved resources go beyond traditional data centers. It includes industrial equipment with sensing and actuation capabilities in addition to the physical computing, storage and network components. The virtualization layer includes the tools and the technologies required to provide a virtualization environment for hosting VNF instances. While the slicing layer refers to the deployed industrial slices in order to accommodate specific industrial machinery's QoS. At this level, we define a set of $L=\left\{l_{j}, \ldots, l_{L}\right\}$ slices based on throughput $R$ (with bandwidth $\lambda_{r}$ ), transmission delay $D$, and urgency factor $\partial$. Each slice is responsible for serving a set of $N=\left\{n_{i}, \ldots, n_{N}\right\}$ assigned IoT devices through a set of 
$G=\left\{g_{k}, \ldots, g_{G}\right\}$ gateways. Where $\theta_{i, j, k}$ denote the binary value that represent the assignment success of devices $i$ to the slice $j$ through gateway $k$. All these layers interact with the general administrative control unit represented in the SDN controller, which can control industrial network in a centralized fashion. By interacting with the NFV MANO (Management and Orchestration), SDN controller is in charge of guaranteeing several industrial QoS constraints. It can acquire and allocate virtual resources for slices and enable industrial network reconfigurability, by exploiting the generated virtual flows from devices, in order to meet dynamically industrial devices QoS changes. Assume that the assigned device $i$ to the slice $j$ generate a virtual flow $f_{i, j, k}$ that goes from the gateway $k$ to the SDN controller and is characterized by a utility metrics $U_{i, j, k}$. SDN controller must define also the slice's request, compute the available resources, and serve slices requirements, in a way that avoids resources starvation. At this level, we denote by $C_{j, k}$ the requested physical resources for the slice $j$ on gateway $k$, while $\zeta_{k}$ is the total gateway capacity.

In this work, we aim to jointly optimize industrial machinery QoS and network energy efficiency by providing dynamically slice members with the requested physical resources.

\section{B. Multi-Objective Optimization Model Formulation}

The purpose of this work is to optimize the performance of the industrial network in term of QoS and energy consumption. Toward this goal, the network slicing optimization concept consists of two main steps. Firstly, is to find the best interslicing resource reservation strategy. This will impose challenges in aggregating necessary parameters, reconfiguring slices, and updating reserved resources. Secondly, is to establish the best intra-slicing resource allocation strategy. This will bring other challenges in reconfiguring devices and updating resource allocation. These problems are formulated into multi-objective functions as follows.

\section{1) QoS Model}

We define the QoS type in each slice based on the Data Rate $R$ and the Transmission Delay $D$. The Data Rate model defined in (1) is denote by the ratio between the peak rate $\chi$ and the slice members $n_{j}$. The peak rate of each IoT device depends on many factors such as the received power $P_{r}$, the transmit power $P_{t}$, and the received SINR, which are written in (2), (3), and (4) respectively [11] [12].

$$
\begin{array}{ll}
R_{i, j, k}=\frac{\chi}{n_{j}} & \text { (1) } P_{r_{i, j}}=\frac{P_{t}}{L_{0}} d^{-\delta} h \\
P_{t_{i, j}}=\frac{\operatorname{SNIR}\left(P_{I}+P_{N}\right)}{L} & \text { (3) } \operatorname{SINR} R_{i, j}=\frac{P_{r}}{P_{I}+P_{N}}
\end{array}
$$

$R_{i, j, k}$ represent the Data Rate for an industrial IoT device $i$ assigned to the slice $j$ on gateway $k$. Where $P_{I}$ and $L$ are respectively the receiver noise and the path loss. $L_{0}$ is a constant, in which depends on the antenna gains and transmission frequency. $h$ denotes the random variable that represents the channel fading. Finally, $d$ and $\delta$ denotes respectively the distance between the trans-receiver and the path loss exponent.
Moreover, the Transmission Delay $D_{i, j, k}$ of a device assigned to the slice $j$, is expressed as in (5). Where $M_{i, j, k}$ is the packet length that is trans-received from a device $i$.

$$
D_{i, j, k}=\frac{M_{i, j, k}}{R_{i, j, k}}
$$

Based on what was previously mentioned, the QoS cost is modeled by (6).

$$
\begin{gathered}
Q o S_{i, j, k}=\overline{R_{i, j, k}}+\left(1-\overline{D_{i, j, k}}\right) \\
\max \sum_{i \in N} Q o S_{i, j, k}, \forall j \in L, k \in G
\end{gathered}
$$

where $Q o S_{i, j, k}$ is the reimbursements that should be maximized at each slice and at each gateway. In addition, $\overline{R_{i, j, k}}$ and $\overline{D_{i, j, k}}$ are adopted as a normalized values that denotes the Throughput and the Transmission Delay achieved by industrial connected device respectively.

\section{2) Energy Consumption Model}

The energy consumption model is considered as the required power to trans-received a data packet, which depends on the received power and the transmitted power given in (2) and (7) respectively. In addition, the connected device consumes a $P_{0}$ power by the communication module. In this context, the active and the sleep mode, as in (8), are the two energy states of an IoT device that need to be considered [11] [12].

$$
\begin{gathered}
P_{t_{i, j}}=\frac{P_{r_{i, j}} L_{0}}{d^{-\delta} h} \\
E_{i, j, k}=\left[\eta P_{\left.t_{i, j}+P_{0}\right]}\right] T_{\text {active }}+\left[\eta P_{\left.t_{i, j}+P_{0}\right] T_{\text {sleep }}}\right. \\
\min \sum_{i \in N} E_{i, j, k}, \forall j \in L, \forall k \in G
\end{gathered}
$$

where $\eta$ denotes the electric-to-RF power conversion factor. $E_{i, j, k}$ represents the energy consumptions for the device $i$ assigned to the slice $j$ that should be minimized for each slice.

Addition to the energy and QoS factors, the Packet Error Rate (PER), formulated in (9), is an interesting factor which reflects the efficiency and reliability of the devices in each slice.

$$
\begin{gathered}
P E R_{i, j, k}=\left(\frac{S_{p}}{T_{p}}\right) \times 100 \\
\min \sum_{i \in N} P E R_{i, j, k}, \forall j \in L, \forall k \in G
\end{gathered}
$$

where $S_{p}$ denotes the number of successful packets and $T_{p}$ is the number of the total packet sent. PER is the other cost function that should be minimized in order to guaranteed efficiency and reliability in each slice.

\section{3) Network Slicing Problem Formulation}

In this paper, network slicing optimization problem consists of three steps. The first one involves the admission and the assignment of devices to the desired slice. The second step is the dynamic inter-slicing resources reservation. While the third step is the intra-slice resource allocations. Firstly, we define slices based on urgency factor, delay, and throughput, and then we look to assign devices to the slice that meets its QoS 
requirements. It is remarkable that the urgency factor, delay and throughput are the key clues for defining the device priority. Furthermore, there are other factors must be taken into consideration such as PER, reliability and the huge load rate of devices to the network. Secondly, we search to estimate the needed inter-slice capacity $C_{j, k}$ based on the required throughput. The purpose of the last step is to optimize the intraslice resources allocation for each slice members. Therefore, the Multi-Objective Optimization for the slicing and the resources allocation problem is formulated in (10).

$$
\min \sum_{i \in N} \theta_{i, j, k} E_{i, j, k} / \operatorname{QoS}_{i, j, k}, \forall j \in L, \forall k \in G
$$

subject to the following constraints:

$$
\begin{aligned}
& \sum_{i \in N} \theta_{i, j, k} R_{i, j, k}\left\langle R_{i, j, k}^{\max }, \forall j \in L, k \in G\right. \\
& \lambda_{r_{j, k}} \cap \lambda_{r_{j^{\prime}, k}}=\varnothing, \forall j \in L, k \in G \\
& 0\left\langleP _ { t _ { i , j , k } } \left\langle P_{t_{i, j, k} \max }^{\text {a }}, \forall j \in L, \forall k \in G, \forall i \in N\right.\right. \\
& \theta_{i, j, k} \in\{0,1\}, \forall j \in L, \forall k \in G, \forall i \in N
\end{aligned}
$$

where constraint (11) guarantees that the sum of transferred traffic by a devices $i$ assigned to the slice $j$ should not exceed the maximum allocated data rate capacity. Moreover, without violating the non-interference principle between slices, constraint (12) ensures a perfect isolation between them. In other words, each slice has its own bandwidth, whether it was reserved on the same gateway or on different gateways. In addition, each device consumes a transmission power to transfer its traffic data, which should not exceed the maximum transmission power; this is provided by constraint (13). Furthermore, the binary assignment value of a device $i$ to the slice $j$ on the gateway $k$, is assured by constraint (14).

\section{THE PROPOSED SLICING APPROACH}

The proposed industrial network slicing-based resource allocation scheme, consists of three main steps. Firstly, by using the Online Gaussian Mixture Model Clustering (OGMMC), each device is assigned to the slice that meets its QoS requirements. At the end of this step, the mean throughput for each slice will be estimated. Secondly, radio resources will be dynamically reserved for each slice based on the dynamic MiniBatch Gradient Descent Algorithm (MBGD). Finally, the preserved radio channels for each slice will be dynamically allocated to the slice members based on the Max-Utility IntraSlice Resource Allocation algorithm.

\section{A. IoT devices Assignment and Mean Throughput Estimation: Online GMM Clustering Algorithm}

Due to the ultra-diversity of industrial 4.0 services, slices are defined based on urgency, energy and efficiency requirements to meet their objectives. Our proposed architecture is composed of three virtual industrial slices. The first slice called "Ultrahigh Critical of Latency and Efficiency (UCLE)" which has the most interest slicing priority and gives more importance to the QoS, efficiency, and reliability. This makes it required by several industrial IoT applications for safety such as emergency action and safeguarding systems. Where "High Critical of
Latency and Efficiency (HCLE)" slice gives a less prominence to the latency and considers reliability as a first target. This service is required by the scale readings applications. The last slice is the "Low Critical of Latency and Efficiency (LCLE)", which has the lowest slice priority with non-guaranteed QoS and efficiency. TABLE. I summarizes the key QoS requirements [13] [14] [15].

TABLE. I. QoS Requirements for Industrial Slices

\begin{tabular}{cccccc}
\hline $\begin{array}{c}\text { Slice } \\
\text { type }\end{array}$ & Latency & Reliability & $\begin{array}{c}\text { Packet } \\
\text { size }\end{array}$ & $\begin{array}{c}\text { Priority } \\
\text { (urgency) }\end{array}$ & Applications \\
\hline UCLE & $50 \mathrm{~ms}$ & $1-10^{(-6)}$ & $24 \mathrm{~B}$ & 1 & $\begin{array}{c}\text { Emergency action, } \\
\text { safeguarding systems }\end{array}$ \\
\hline HCLE & $100 \mathrm{~ms}$ & $1-10^{(-6)}$ & $512 \mathrm{~B}$ & 2 & Scale readings \\
\hline LCLE & $500 \mathrm{~ms}$ & $\gg>1-10^{(-6)}$ & $250 \mathrm{~B}$ & 3 & $\begin{array}{c}\text { Standard mobile } \\
\text { robot }\end{array}$ \\
\hline
\end{tabular}

After specifying each slice requirements, IoT devices will be assigned to the corresponding slices. In this context, GMM is adopted as a dynamic and online clustering method (OGMMC) to assign devices to the desired slice, by checking its QoS demands and estimating the mean throughput for slices [16].

Considering a set of $J$ mixture multivariate Gaussian Distributions, indexed by the set of parameters $\Theta=\left\{\alpha_{j}, \theta_{j}\right\}$, where $\theta_{j}=\left\{\mu_{j}, \Sigma_{j}\right\}$ denotes the parameters of the $j^{\text {th }}$ Gaussian distribution, in which the mean is denoted by $\mu_{j}, \quad \sum_{j}$ is the covariance, and $\alpha_{j} \in\{0,1\}$ is the mixing probabilities. Assume that all devices data point $\left\{n_{i}, \ldots, n_{N}\right\}$ are independently and identically distributed according to the mixture probability density function $P\left(n_{i} \mid \theta_{j}\right) . \Theta$ is the parameter that will be estimated to clusters using the Maximum Likelihood Estimation (MLE) process, as in (15). While the log-likelihood is formulated in (16).

$$
\begin{aligned}
& L(\Theta \mid N)=\prod_{i \subset N} P\left(n_{i} \mid \theta_{j}\right)=\prod_{i \subset N}\left(\sum_{j \subset J} \alpha_{j} P_{j}\left(n_{i} \mid \mu_{j}, \sum_{j}\right)\right) \\
& \log (\Theta \mid N)=\sum_{i \subset N} \log \left(\sum_{j \subset J} \alpha_{j} P_{j}\left(n_{i} \mid \mu_{j}, \Sigma_{j}\right)\right)
\end{aligned}
$$

In view of the structural complexity of (16), the optimal $\Theta$ cannot be obtained by setting the derivatives to zero. Expectation Maximization (EM) process [17], is a powerful method used to maximize the log-likelihood function and find the optimal parameters. The latter updates iteratively the parameters of individual Gaussian distributions. The given data are considered as incomplete data. This allows defining $M$ latent variables $M=\left\{m_{i}, \ldots, m_{M}\right\}$ where each $m_{i}$ indicates which Gaussian component generates the data vector $n_{i}$. The new function that should be maximized is formulated in (17).

$$
\begin{gathered}
\Psi(\Theta, \varphi)=\sum_{i \subset N} \sum_{j \subset J} \log \left(\alpha_{j} P_{j}\left(n_{i} \mid \theta_{j}\right)\right)- \\
\sum_{i \subset N} \sum_{j \subset J} \omega_{i, j} \log \left(\omega_{i, j}\right)
\end{gathered}
$$

where $\varphi=\left\{\omega_{i, j}\right\}$ is the assignment of data vectors (devices) to the clusters. In the expectation step, EM process tends to calculate the probability of a device $i$ belongs to the cluster $j$ (slice), by maximizing the $\Psi$ function over the assignment $\varphi$ and by considering the posterior probability $\omega_{i, j}$ formulated in (18). 


$$
\omega_{i, j}=P\left(m_{i}=j \mid n_{i}, \Theta\right)=\frac{\alpha_{j} P_{j}\left(n_{i} \mid \theta_{j}\right)}{\sum_{j \subset J} \alpha_{j} P_{j}\left(n_{i} \mid \theta_{j}\right)}
$$

After that, a maximization of $\Psi$ function over the parameter $\Theta$ will be in the maximization step. As a maximization result, the parameters $\Theta$ will be estimated and updated iteratively until the convergence. The updated mean, mixing probabilities and covariance are presented respectively in (19), (20), and (21).

$$
\begin{aligned}
& \hat{\mu}_{j}^{\text {new }}=\frac{\sum_{i \subset N} \omega_{i, j} n_{i}}{\sum_{i \subset N} \omega_{i, j}} \quad \text { (19) } \quad \hat{\alpha}_{j}^{\text {new }}=\frac{1}{n} \sum_{i \subset N} \omega_{i, j} \\
& \widehat{\sum}_{j}^{\text {new }}=\frac{\sum_{i \subset N} \omega_{i, j}\left(n_{i}-\widehat{\alpha}_{j}^{\text {new }}\right)\left(n_{i}-\widehat{\alpha}_{j}^{\text {new }}\right)^{T}}{\sum_{i \subset N} \omega_{i, j}}
\end{aligned}
$$

The optimum slicing strategy for a limited physical capacity, is to virtually reserve resources for each slice based on the mean throughput $R_{j}^{T}$ of its members. After the assignment step, OGMMC calculates $R_{j}^{T}$ for slices, as in (22), with respect to the urgency factor.

$$
R_{j}^{T}=\frac{\sum_{i \subset N} R_{i, j, k}}{n_{i, j}}, \forall j \in L, \forall k \in G
$$

Each new device sends a connection request to the server that will set up the flag $H$, in which the Online GMMC algorithm, as in Pseudo-Code 1, will be re-executed.

\section{B. Dynamic Inter-Slicing Resources Reservation: Dynamic MBGD Algorithm}

After assigning IoT devices to the slice that meets its QoS requirements and estimating the mean throughput for slices, we seek in this section to reserve dynamically inter-slices channel resources. To reach this goal, MBGD [18] learning algorithm is adopted as a powerful scheme to improve QoS and minimize cost, in (10), by finding the optimal throughput parameter needed to split channel resources between slices.

In the context of Industrial IoT (IIoT), we assume that slices should be ready to support and serve IoT devices. The global idea is to reserve a minimum capacity level $C_{j, k}$ for slices . Then, by checking slices requirements and learning dynamically devices throughput, more radio resources will be reserved for slices.

The adopted MBGD scheme consists of two phases; prelearning phase and learning phase. In the pre-learning phase (line 1 to 7 in the Pseudo-Code 2), MBGD splits physical resources between slices, by reserving a minimum radio channels based on the estimated mean throughput, even there is no assigned devices (line 4 to 6 ). With respect to the slice urgency factor, it starts by computing the slice rate $\gamma_{j}$ based on $R_{j}^{T}$, as in (23). This is for defining an appropriate and optimal resource distribution strategy and for not exceeding the maximum gateway capacity. Then, the defined level of physical resources will be reserved, as in (24). At the end of this phase, the process computes the unserved capacity $\xi_{k}$ on each gateway, as formulated in (25).

$$
\begin{gathered}
\gamma_{j}=\frac{R_{j}^{T}}{\sum_{j \subset L} R_{j}^{T}} \quad \text { (23) } \quad C_{j, k}=\gamma_{j} * \varsigma_{k}, \forall j \in L, \forall k \in G \\
\xi_{k}=\varsigma_{k}-\sum_{j \subset L} C_{j, k}, \forall j \in L, \forall k \in G
\end{gathered}
$$

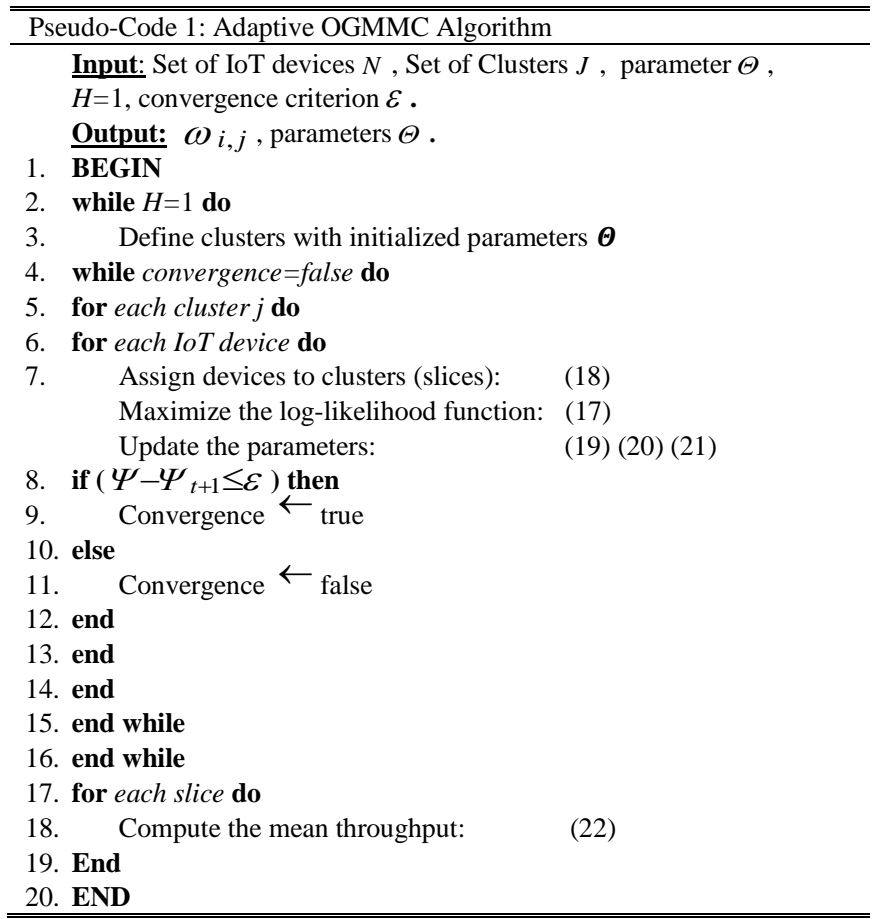

Noting that the slice capacity $C_{j, k}$ do not exceed the maximum capacity $\zeta_{k}$ provided by each gateway. That is to say, that the mean throughput $R_{j}^{T}$ should not exceed the sum of requested throughput for all IoT devices. Therefore, (22) and (23) must satisfy constraints (27) and (26) respectively.

$$
0<\gamma_{j}<1 \quad \text { (26) } \quad 0<R_{j}^{T}<\sum_{j \subset L} R_{j}^{T}
$$

After defining a minimum capacity level for each slice, MBGD acts as a brain in each gateway. It learns QoS and energy consumption properties in order to find the best configuration parameter that meets slice demands. This can be done by tracking dynamically slices member's throughput requirements and updates its radio resources capacity.

The common chosen parameter in the learning phase (line 8 to 17), is $R_{i, j, k}$ that can interact with QoS, energy consumption and PER. However, when the throughput increase, QoS will be maximized. This refer to the capacity that will be increased according to $R_{i, j, k}$ that decrease the transmission delay as denoted in (5). On the other hand, the activation time $T_{\text {active }}$ of the IoT device will decrease because of the higher speed transmission packets in a large bandwidth. The latter has an effect on energy consumption by decreasing the transmission power and give more chance to increase the percentage of successful transmitted packet. This parameter will be tuned and configured online, using the Mean Square Error (MSE) process, based on the other discussed parameters (training set). In this context, let denote by $\mathbb{C}$ the current observation values and $\mathbb{C}^{\prime}$ is the trained output value formulated in (28), where $v$ is the learning rate. The MSE function is defined in (29). Where $\delta_{j}$ is the Mini-Batch size and $b_{i, j, k}=\left(1-\overline{D_{i, j, k}}\right)$.

$$
\mathbb{C}^{\prime}=E_{i, j, k} / \operatorname{QoS}_{i, j, k}
$$




$$
\begin{aligned}
M S E=\frac{1}{2 \delta_{j}} \sum_{i \rightarrow \delta_{j}}\left(\mathbb{C}^{\prime}-\mathbb{C}\right)^{2} & = \\
& \frac{1}{2 \delta_{j}} \sum_{i \rightarrow \delta_{j}}\left(\frac{E_{i, j, k}}{\overline{R_{i, j, k}+b_{i, j, k}}}-\mathbb{C}\right)^{2}
\end{aligned}
$$

MBGD repeatedly iterates through the training set (power, throughput, and delay) and update the parameter $R_{i, j, k}$ according to the gradient error respectively in (31) and (30).

$$
\begin{gathered}
\nabla_{R}=\frac{-1}{\delta_{j}} \sum_{i \rightarrow \delta_{j}} \frac{E_{i, j, k}}{\left(\overline{R_{i, j, k}}+b_{i, j, k}\right)^{2}} *\left(\frac{E_{i, j, k}}{\left(\overline{R_{i, j, k}}+b_{i, j, k}\right)}-\mathbb{C}\right) \\
\frac{R_{i, j, k}^{n e w}}{=} \overline{R_{i, j, k}}-v \nabla_{R}
\end{gathered}
$$

Thereafter, MBGD checks resources demands for each slice and updates slice rate and requested capacity, formulated respectively in (32) and (33). Then, it updates reserved and unserved capacity presented respectively by formulas (34) and (35). As summarized in Pseudo-Code 2, the learning phase will be repeated (line 8 to 17) until it serves all slices requirements and stops when there are no more resources to serve it or convergence is reached. We note that, notations with "new" means the predicted value by the proposed learning process. $C_{j, k}^{a d d}$ denote the new requested capacity to be added, $C_{j, k}^{\text {new }}$ is the total reserved capacity, $\xi_{j, k}^{\text {new }}$ is the new unserved capacity, while $R_{i, j, k}$ denote the current observation parameter.

$$
\begin{aligned}
& \gamma_{j}^{\text {new }}=\frac{\overline{R_{i, j, k}^{n e w}}}{\sum_{j \subset L} \overline{R_{i, j, k}}} \\
& C_{j, k}^{\text {new }}=C_{j, k}+C_{j, k}^{a d d}
\end{aligned}
$$

$$
\begin{gathered}
C_{j, k}^{a d d}=\gamma_{j}^{n e w} * \varsigma_{k} \\
\xi_{j, k}^{\text {new }}=\varsigma_{k}-\sum_{j \subset L} C_{j, k}^{\text {new }}
\end{gathered}
$$

\section{Dynamic Intra-Slicing Resources Allocation: Max-Utility algorithm}

After inter-slice resources reservation, we seek in this stage to improve and optimize intra-slice resources allocation. The latter is reached by maximizing utility metric $U_{i, j, k}$ for IIoT devices, in each slice and on each gateway.

Utility metric is modeled based on reliability weight $\left(w_{r}\right)$ and load weight $\left(w_{l d}\right)$. As mentioned previously in TABLE. I, slices are different in term of QoS. However, utility metrics can be expressed as follow:

$$
\begin{gathered}
U_{U C L E}=x_{r}\left(w_{r} \vartheta_{r}\right), x_{r}=\operatorname{SINR}_{i, j, k} / \operatorname{SINR}_{\max }, x_{r} \in\{0,1\} \\
U_{H C L E}=w_{r} \vartheta_{r}+w_{l d} \vartheta_{l d} \\
U_{L C L E}=w_{l d} \vartheta_{l d}
\end{gathered}
$$

where the highest required reliability and urgency for UCLE slice, is denoted by (36). $x_{r}$ is considered as a minimum threshold guaranteed during search for the highest reliable link. $\vartheta_{l d}$ and $\vartheta_{r}$ are respectively the load rate and the reliability rate. The algorithm summarized in Pseudo-Code 3 based on the Analytical and Hierarchy Process (AHP), searches for the efficient and reliable link that gives the highest utility metric and allocate resources accordingly [19].

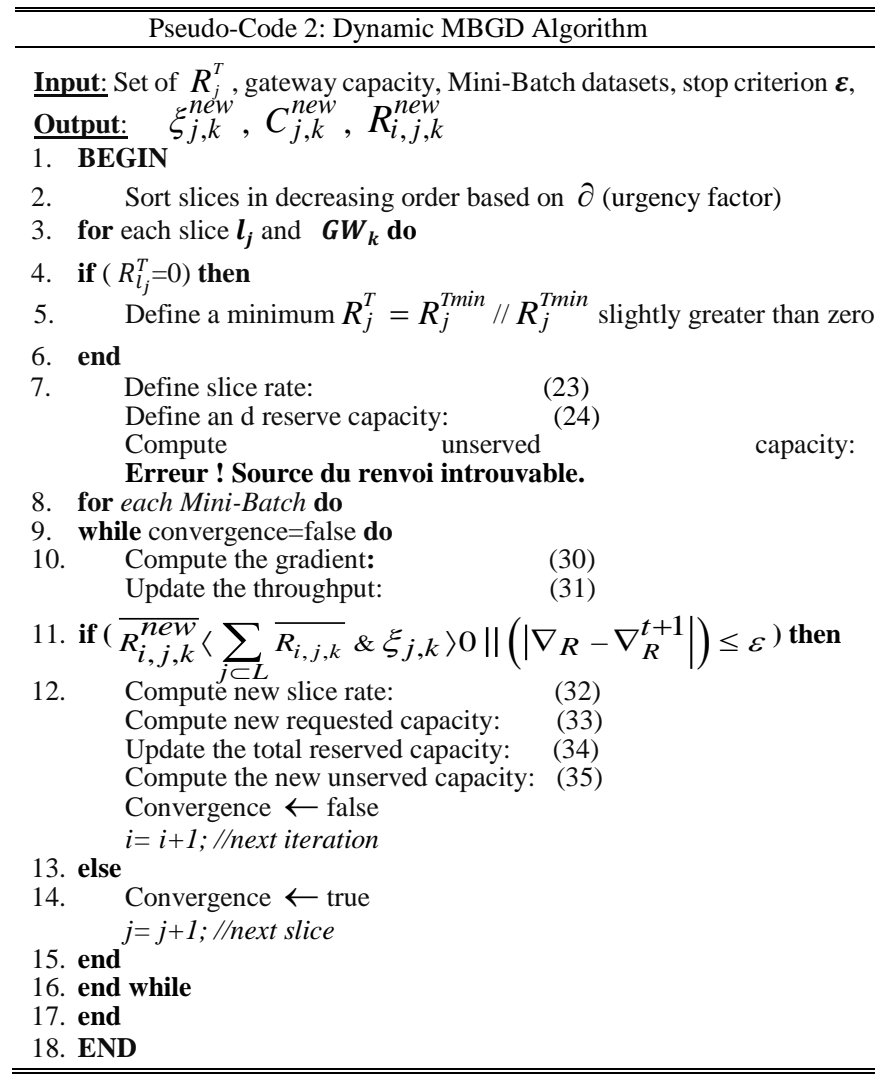

Formula (37) represent the trade-off between reliability and load which explains the less critical latency and priority of HCLE slice and the massive number of connected IoT devices. In this case, the process goes to find the optimal link that gives a perfect reliability with minimum load. While LCLE slice modeled by (38), shows the non-guaranteed latency and QoS requirements. Here, the adopted algorithm seeks to find the virtual link without considering reliability.

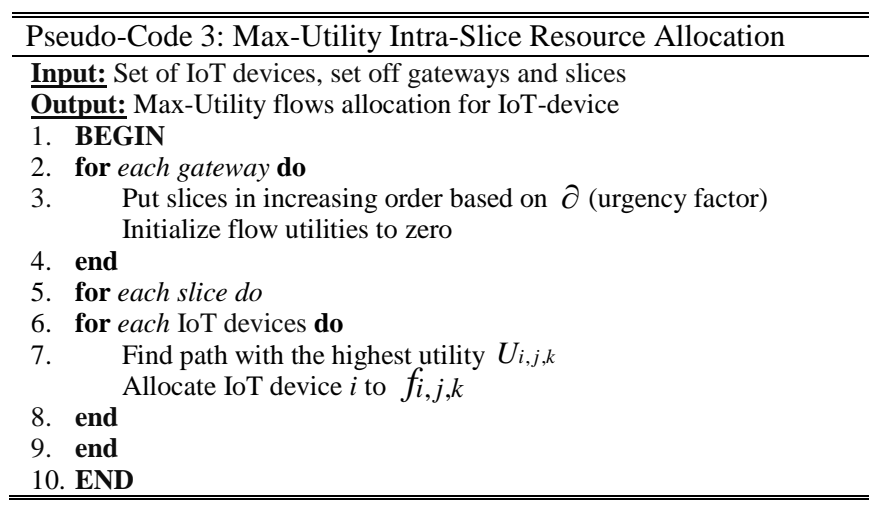

In general case, we consider a set on IoT device denoted as a source node assigned to slice $j$, uploads traffic through gateways $k$. The goal is to find the efficient virtual flow $f_{i, j, k}$ that maximize device utility metric $U_{i, j, k}$, as in (39), in order to allocate efficiently resources .

$$
U_{i, j, k}=U_{i, j, k}^{\prime}+U_{i, j, k}^{\prime \prime}
$$

where $U_{i, j, k}^{\prime \prime}$ and $U_{i, j, k}^{\prime}$ are the utilities provided by each gateway, in which depends on reliability and load. 


\section{Simulation AND Results ANALYsis}

In this section, we study the performance of the proposed approach and deeply analyze results. The suggested scheme is implemented in NS3 simulator [20]. TABLE. II summarizes simulation parameters.

TABLE. II. Simulation parameters

\begin{tabular}{lc}
\hline \hline \multicolumn{1}{c}{ Parameter } & Value \\
\hline \hline Simulation area & $1 \mathrm{~km}^{2}$ \\
Power consumption Tx/Rx & $25 \mathrm{mw}$ \\
Battery capacity & $230 \mathrm{mAh}$ \\
Number of Nodes & 1000 \\
Number of gateways (GWs) & 1 \\
Number of channels & 8 per GW \\
Used protocol & LoRaWAN \\
\hline \hline
\end{tabular}

We consider a set of IoT devices initialized with 100 devices, in which increased till it reaches 1000 in a single gateway. They are distributed randomly in an industrial area of one square kilometer. We implement firstly the Slicing Methodology Configuration (SMC). Then, Static Configuration (SC) will be implemented. The latter has the same simulation parameters to the SMC, but it does not contain the QoS constraint. The objective is to study the QoS profitability for slicing strategy results and make a comparison with the traditional configuration in term of Energy Consumption (EC), Transmission Delay (TD), and PER.

\section{A. Energy Consumption Analysis}

We assume that the sleep power for IoT devices is set to zero. As presented in Figure 2 (a), the total energy consumption for each slice depends on the number of assigned devices and QoS configuration.

As in TABLE. I, LCLE slice is configured with the lowest priority and nonguaranteed QoS with considering load only. As results, a large number of devices, will be assigned to this slice, in which activation time will be increased proportionally. These leads to increase the power consumption. While HCLE slice scored less power consumption. The latter is configured with a less critical latency but also with a guaranteed reliability and efficiency. As result, a little set of devices will respect this constraint and assigned to HCLE. The tradeoff between reliability and less critical latency leads to minimizing energy consumption compared to the previous slice. While configuration in UCLE slice leads to the most efficient power consumption compared to the others. This return to the inter and intra process that gives higher importance and priority for QoS configuration, and reliability in Utility calculation. It allows the little set of assigned devices to take the most reliable gateway with smaller duration of spectrum occupation time.

For more evaluation, the mean EC for proposed scheme was compared to the EC for the SC. As denoted in Figure 2 (b), the $\mathrm{EC}$ for static method increases exponentially while number of deployed devices increases. This because that all IoT devices are assigned to the same network without respecting reliability, QoS, and efficiency constraint. This prove the efficiency of the proposed slicing method.
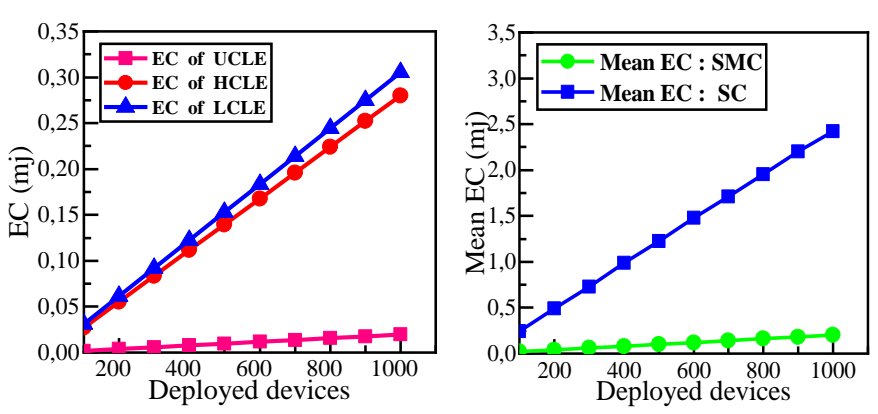

Figure 2: Energy Consumption evaluation

\section{B. Delay Variation Analysis}

Relying on QoS class in TABLE. I, each slice is configured with packet size constraint. It is remarkable that HCLE has the highest packet size than LCLE and UCLE slices. While UCLE slice is configured with the highest reliability and efficiency. This will give mores chance to UCLE slice to serves its members, by maximizing utility metrics and allocating more radio channels. As results, throughput will be increased, allowing delay to be reduced to a minimum. This increases the percentage of devices that have not violated their delay threshold. This is not the case for HCLE, which is configured with medium priority, large packet size, and a utility depending on reliability and load. As the number of deployed devices increases, delay will increase exponentially, even if the learning process reserves more resources. Thus, increase the percentage of IoT devices that violated its delay threshold. Instead, delay in LCLE will be increased according to the increase of its member but it remains less than HCLE. This refers to the QoS constraint that configure LCLE with a little packet size than HCLE and consider the load only. Figure 3 (a) demonstrates the delay variation for slices, while Figure 3 (b) demonstrates the percentage of served devices in delay.
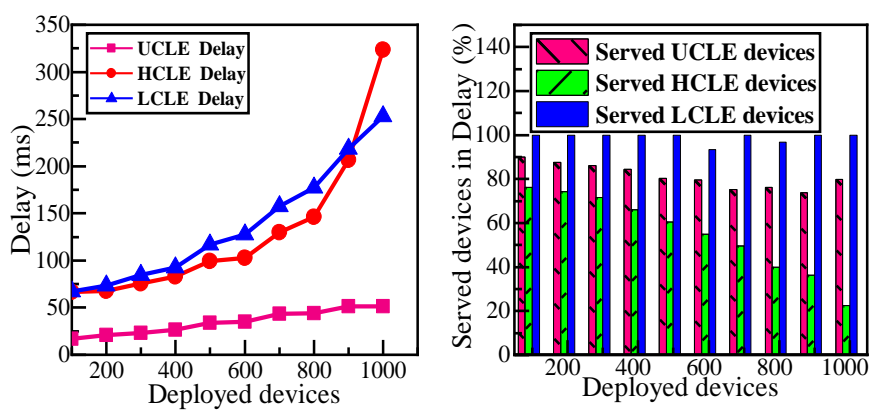

Figure 3: Delay variation and percentage of Served devices

More performances evaluations were conducted, and the proposed slicing method was compared to the static method in term of delay variation and percentage of unserved devices in delay. As seen in Figure 4 (a) and (b), SC had the worst results with highest delay varies exponentially with the increase of deployed devices number. Also $88 \%$ of devices did not respect their delay thresholds on 1000 deployed devices compared to $32 \%$ of the SMC. This refers to the random configuration that did not take into consideration QoS requirements of devices. 

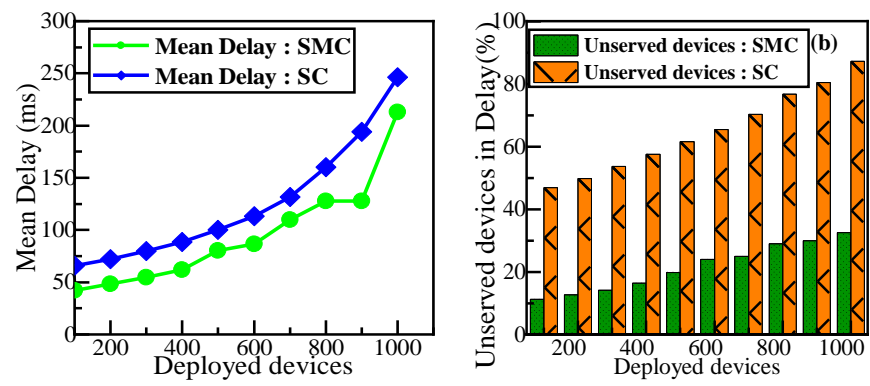

Figure 4: Mean Delay variation and percentage of Unserved devices

\section{Packet Error Rate Analysis}

Figure 5 (a), shows the evolution of PER in each slice. As described previously, the proposed process gives more importance to the UCLE slice, by checking its QoS demands and serve it. Then, it moves to the HCLE slice, and later if there are unserved resources it can reserve some to LCLE slice. As results, UCLE slice will be frequently served, and may therefore limit PER of its members. It is remarkable that PER in UCLE increase when devices increase at 200 and 300, then PER decrease at 400 devices, etc. This returns to the learning tools that try to avoid resource starvation in each slice and dynamically reserves channels following throughput demands. As the deployment device increases, congestion increases, and reserved resources will no longer be sufficient to support devices requirements. At this stage, PER will be increase until process serve its demand in future iteration. In fact, it is the same thing for the other two slices, but with considering less QoS constraint to HCLE and no QoS constraint to the LCLE slice. This implies that fewer radio channels will be reserved, in which congestion will be increased, while PER will also increase. By the other hand, with static configuration, PER was highly increased. This refers to the continuously increased congestion with the increase of deployment devices. This because the QoS configuration is not considered. Results in Figure 5 (b) prove the efficiency of the optimization process in reducing PER with $40 \%$ compared to $20 \%$ of the SC.
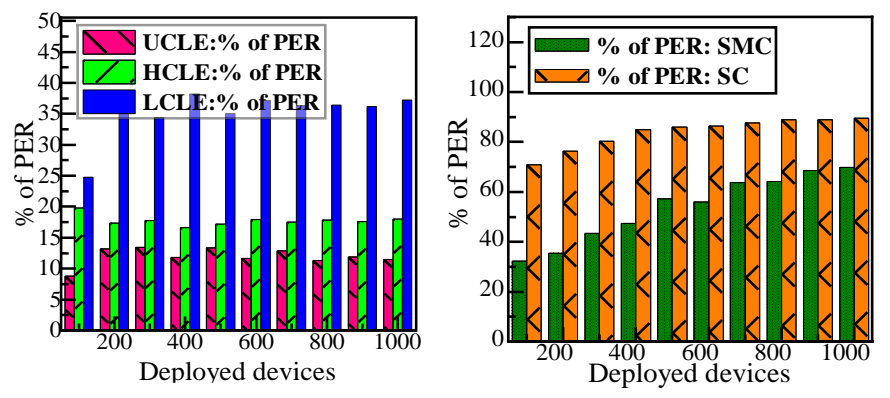

Figure 5: Percentage of PER evaluation for both configurations

\section{CONCLUSION}

The ever-increasing exploitation of smart devices with improved capabilities, is leading to a radical change in the industrial landscape. However, low latency, reliability, and efficiency are required to support new Industrial 4.0 challenges. Network slicing paradigm benefits can be extended to address outrange performance requirements. In this context, we have proposed industrial network slicing framework based on machine learning tools in order to meet QoS requirements online and dynamically over the industrial network. Firstly,
OGMMC was proposed to assign devices to the desired slice that meet its QoS demands and to estimate mean throughput slices requirements. Secondly, Mini-Batch based slicing scheme was proposed to reserve dynamically radio channels to slices. Finally, steps Max-Utility algorithm was adopted in order to efficient allocate the gateway resources to the slice's members. Simulations results show the efficiency of the proposed slicing method compared to the static method in saving energy consumption, reducing delay, and PER.

\section{REFERENCES}

[1] D. Hanes, G. Salgueiro, P. Grossetete, R. Barton, and J. Henry. (2017). IoT Fundamentals: Networking Technologies, Protocols, and Use Cases for the Internet of Things. Cisco Press.

[2] A. Colombo, W. Karnouskos, S. Kaynak, O. Y. Shi, and S. Yin. (2017). Industrial cyberphysical systems: A backbone of the fourth industrial revolution. IEEE Industrial Electronics Magazine, 11(1), pp. 6-16.

[3] I. Afolabi, T. Taleb, K. Samdanis, A. Ksentini, and H. Flinck. (2018). Network slicing and softwarization: A survey on principles, enabling technologies, and solutions. IEEE Communications Surveys \& Tutorials, 20(3), pp. 2429-2453.

[4] D. Li, M. T. Zhou, P. Zeng, M. Yang, Y. Zhang, and H. Yu. (2016). Green and reliable software-defined industrial networks. IEEE Communications Magazine, 54(10), pp. 30-37.

[5] H. Zhang, C. Jiang, N. C. Beaulieu, X. Chu, X. Wen, and M. Tao. (2014, July). Resource allocation in spectrum-sharing OFDMA femtocells with heterogeneous services. IEEE Transactions on Communications, 62(7), pp. 2366-2377.

[6] V. Yazici, U. C. Kozat, and M. O. Sunay. (2014, Nov). A New Control Plane for 5G Network Architecture with a Case Study on Unified Handoff, Mobility, and Routing Management. IEEE Commun. Mag, 52(11), pp. 76-85.

[7] Q. Li, G. Wu, A. Papathanassiou, and U. Mukherjee. (2016). An end-toend network slicing framework for $5 \mathrm{G}$ wireless communication systems. arXiv preprint arXiv:1608.00572.

[8] E. Molina, and E. Jacob. (2018). Software-defined networking in cyberphysical systems: A survey. Computers \& Electrical Engineering, 66, pp. 407-419

[9] J. Wan, S. Tang, Z. Shu, D. Li, S. Wang, M. Imran, and A. V. Vasilakos. (2016). Software-defined industrial internet of things in the context of industry 4.0. IEEE Sensors Journal, 16(20), pp. 7373-7380.

[10] J. Wan, C. F. Lai, H. Song, M. Imran, and D. Jia. (2019). SoftwareDefined Industrial Internet of Things. Wireless Communications and Mobile Computing.

[11] B. Al Homssi, A. Ai-Hourani, K. G. Chavez, S. Chandrasekharan, and S. Kandeepan. (2018, December). Energy-Efficient IoT for 5G: A Framework for Adaptive Power and Rate Control. 12th International Conference on Signal Processing and Communication Systems (ICSPCS), IEEE, pp. 1-6.

[12] A. Al-Hourani, S. Kandeepan, and E. Hossain. (2016). Relay-assisted device-to-device communication: A stochastic analysis of energy saving. IEEE Transactions on Mobile Computing, 15(12), pp. 3129-3141.

[13] A. E. Kalør, R. Guillaume, J. J. Nielsen, A. Mueller, and P. Popovski. (2018). Network slicing in industry 4.0 applications: Abstraction methods and end-to-end analysis. IEEE Transactions on Industrial Informatics, 14(12), pp. 5419-5427.

[14] M. Lucas-Estañ, M. Sepulcre, T. Raptis, A. Passarella, and M. Conti. (2018). Emerging Trends in Hybrid Wireless Communication and Data Management for the Industry 4.0. Electronics, 7(12), pp. 400.

[15] A. E. Kalør, R. Guillaume, J. J. Nielsen, A. Mueller, and P. Popovski. (2017). Network slicing for ultra-reliable low latency communication in industry 4.0 scenarios. arXiv preprint arXiv:1708.09132.

[16] G. Celeux, and G. Govaert. (1995). Gaussian parsimonious clustering models. Pattern recognition, 28(5), pp. 781-793.

[17] T. K. Moon. (1996). The expectation-maximization algorithm. IEEE Signal processing magazine, 13(6), pp. 47-60.

[18] S. Ruder. (2016). An overview of gradient descent optimization algorithms. arXiv preprint arXiv:1609.04747.

[19] S. Dawaliby, A. Bradai, and Y. Pousset, (2019). Adaptive dynamic network slicing in LoRa networks. Future Generation Computer Systems, 98, pp. 697-707.

[20] G. Carneiro. (2010, April). NS-3: Network simulator 3. In UTM Lab Meeting, 20, pp. 4-5. 